\title{
Quantum singularities in a model of $f(R)$ gravity
}

\author{
O. Gurtug* and T. Tahamtan \\ Department of Physics, Eastern Mediterranean University, \\ G. Magusa, north Cyprus, Mersin 10, Turkey.
}

\begin{abstract}
The formation of a naked singularity in a model of $f(R)$ gravity having as source a linear electromagnetic field is considered in view of quantum mechanics. Quantum test fields obeying the Klein-Gordon, Dirac and Maxwell equations are used to probe the classical timelike naked singularity developed at $r=0$. We prove that the spatial derivative operator of the fields fails to be essentially self-adjoint. As a result, the classical timelike naked singularity remains quantum mechanically singular when it is probed with quantum fields having different spin structures.
\end{abstract}

\section{INTRODUCTION}

In the last decade, there have been extensive studies in Extended Theories of Gravity (ETG) such as the Lovelock and $f(R)$ gravity theories. The main motivation to study the ETG is to understand the accelerated expansion of the universe and the issue of dark matter/energy (see [1] and references therein for a general review). One of the most attractive branches of the ETG is the $f(R)$ gravity theory in which the standard Einstein's gravity is extended with an arbitrary function of the Ricci scalar $R$ instead of the linear one [1]. In this model, the Ricci scalar $R$ in the Einstein-Hilbert action is replaced with $f(R)=R+\alpha g(R)$, where $g(R)$ is an arbitrary function of $R$ so that in the limit $\alpha=0$, one recovers the Einstein limit. Although the majority of researchers prefer to use this ansatz, in general, finding an exact analytic solution to the field equations is not an easy task. As far as analytic exact solutions are concerned, static, spherically symmetric models in $f(R)$ gravity have been shown to serve for this purpose [2-6]. In this context of static, spherically symmetric solutions of $f(R)$ gravity, the solutions admitting black holes have attracted much attention.

In the context of static, spherically symmetric $f(R)$ gravity, it has recently been shown that [], an exact analytic solution is also possible if one assumes $f(R)$ to have the form of $f(R)=\xi\left(R+R_{1}\right)+2 \alpha \sqrt{R+R_{0}}$, in which $\xi, \alpha, R_{0}$ and $R_{1}$ are constants, a priority to secure the Einstein limit by setting the constants $R_{0}=R_{1}=\alpha=0$ and $\xi=1$. In this model of $f(R)$ gravity, exact solutions with external electromagnetic sources (both linear and nonlinear) are found. It was shown that the solution with a linear electromagnetic field does not admit a black hole while the solution with a nonlinear electromagnetic source admits a black hole solution. The physical properties of the latter solution are investigated by calculating thermodynamic quantities and it was shown to satisfy the first law of thermodynamics. The solution having as a source a linear electromagnetic field resulted with a naked curvature singularity at $r=0$, which is a typical central singularity peculiar to spherically symmetric systems. The solution given in [7], is a kind of extension of a global monopole solution [8] which represents a solution of the Einstein's equations with spherical symmetry with matter that extends to infinity. It can also be interpreted as a cloud of cosmic strings with spherical symmetry [9]. Hence, the spacetime is conical. However, with the inclusion of a linear or nonlinear electromagnetic field, the spacetime is no more conical in the context of $f(R)$ gravity.

Within the framework of ETG gravity, black hole solutions have been widely studied in the literature (see [1, 10] and references therein for a complete review). However, the solutions that result with naked singularities have not been studied in detail. In physics, naked singularities are considered to be a threat to the cosmic censorship hypothesis. Furthermore, as in classical general relativity, compared to the black hole solutions, naked singularities are not well understood in the context of $f(R)$ gravity. This still remains a fundamental problem in general relativity as well as in ETG to be solved. Another important difficulty in resolving this problem is the scale on which the curvature singularity occurs. On these small scales, it is believed that the classical methods should be replaced with quantum techniques in resolving the singularity problems that necessitate the use of quantum gravity. Since the quantum theory of gravity is still "under construction", an alternative method is proposed by Wald 11] which was further developed by Horowitz and Marolf (HM) [12] in determining the character of classically singular spacetime and to see if quantum effects have any chance to heal or regularize the dynamics and restore the predictability if the singularity is probed with quantum particles/fields.

*Electronic address: ozay.gurtug@emu.edu.tr

†Electronic address: tayabeh.tahamtan@emu.edu.tr 
In this paper, we investigate the occurrence of naked singularities in the context of $f(R)$ gravity from the point of view of quantum mechanics. We believe that this will be the unique example wherein the formation of a classically naked curvature singularities in $f(R)$ gravity will be probed with quantum fields/particles that obey the Klein-Gordon, Dirac and Maxwell equations. The criterion proposed by HM will be used in this study to investigate the occurrence of naked singularities.

This criterion has been used successfully for other spacetimes to check whether the classically singular spacetimes are quantum mechanically regular or not. As an example; negative mass Schwarzschild spacetime, charged dilatonic black hole spacetime and fundamental string spacetimes are considered in [12]. An alternative function space, namely the Sobelov space instead of the Hilbert space, has been introduced in [13], for analyzing the singularities within the framework of quantum mechanics. Helliwell and Konkowski have studied quasiregular [14], Gal'tsov-Letelier-Tod spacetime [15], Levi-Civita spacetimes [16, 17], and recently, they have also considered conformally static spacetimes [18]. Pitelli and Letelier have studied spherical and cylindrical topological defects [19], Banados-Teitelboim-Zanelli (BTZ) spacetimes [20], the global monopole spacetime [21] and cosmological spacetimes [22]. Quantum singularities in matter coupled $2+1$ dimensional black hole spacetimes are considered in 23]. Quantum singularities are also considered in Lovelock theory 24] and linear dilaton black hole spacetimes [25]. Recently, the occurrence of naked singularities in a $2+1$ dimensional magnetically charged solution in Einstein-Power-Maxwell theory have also been considered [26].

The main theme in these studies is to understand whether these classically singular spacetimes turn out to be quantum mechanically regular if they are probed with quantum fields rather than classical particles.

The solution to be investigated in this paper is a kind of $f(R)$ gravity extension of the analysis presented in [21] for the global monopole spacetime. The inclusion of the linear Maxwell field within the context of $f(R)$ gravity affects the topology significantly and removes the conical nature at infinity. Furthermore, the true timelike naked curvature singularity is created at $r=0$ which is peculiar to spherically symmetric systems. We investigate this singularity within the framework of quantum mechanics by employing three different quantum fields/particles obeying the Klein-Gordon, Dirac and Maxwell fields with different spin structures.

The paper is organized as follows: In Sec.II, we review the solution found recently in [7], and give the structure of the spacetime. In Sec.III, first, the definition of quantum singularity for static spacetimes is briefly introduced. Then, the quantum fields obeying the Klein-Gordon, Dirac and Maxwell equations are used to probe the singularity. The paper ends with a conclusion in Sec. IV.

\section{THE METRIC FOR $f(R)$ GRAVITY COUPLED TO MAXWELL FIELDS AND SPACETIME STRUCTURE}

Recently, an exact analytic solution for $f(R)$ gravity coupled with linear and nonlinear Maxwell field in four dimensions has been presented in [7]. The corresponding action for $f(R)$ gravity coupled with linear Maxwell field in four dimensions is given by,

$$
S=\int d^{4} x \sqrt{-g}\left[\frac{f(R)}{2 \kappa}-\frac{1}{4 \pi} F\right],
$$

in which $f(R)$ is a real function of the Ricci scalar $R$, and $F=\frac{1}{4} F_{\mu \nu} F^{\mu \nu}$ is the Maxwell invariant. The Maxwell two-form is given by

$$
\mathbf{F}=\frac{Q}{r^{2}} d t \wedge d r+P \sin \theta d \theta \wedge d \varphi
$$

in which $Q$ and $P$ are the electric and magnetic charges, respectively. The static spherically symmetric metric ansatz is

$$
d s^{2}=-B(r) d t^{2}+\frac{d r^{2}}{B(r)}+r^{2}\left(d \theta^{2}+\sin ^{2} \theta d \varphi^{2}\right)
$$

where $B(r)$ stands for the only metric function to be found. The Maxwell equations (i.e. $d F=0=d^{*} F$ ) are satisfied and the field equations are given by

$$
f_{R} R_{\mu}^{\nu}+\left(\square f_{R}-\frac{1}{2} f\right) \delta_{\mu}^{\nu}-\nabla^{\nu} \nabla_{\mu} f_{R}=\kappa T_{\mu}^{\nu},
$$


in which

$$
\begin{aligned}
f_{R} & =\frac{d f(R)}{d R} \\
\square f_{R} & =\frac{1}{\sqrt{-g}} \partial_{\mu}\left(\sqrt{-g} \partial^{\mu}\right) f_{R} \\
\nabla^{\nu} \nabla_{\mu} f_{R} & =g^{\alpha \nu}\left[\left(f_{R}\right)_{, \mu, \alpha}-\Gamma_{\mu \alpha}^{m}\left(f_{R}\right)_{, m}\right]
\end{aligned}
$$

while the energy momentum tensor is

$$
4 \pi T_{\mu}^{\nu}=-F \delta_{\mu}^{\nu}+F_{\mu \lambda} F^{\nu \lambda}
$$

Furthermore, the trace of the field equation (4) reads

$$
f_{R} R+(d-1) \square f_{R}-\frac{d}{2} f=\kappa T,
$$

with $T=T_{\mu}^{\mu}$. The non-zero energy momentum tensor components are

$$
T_{\mu}^{\nu}=\frac{P^{2}+Q^{2}}{8 \pi r^{4}} \operatorname{diag}[-1,-1,1,1],
$$

and with zero trace, we have

$$
f=\frac{1}{2} f_{R} R+3 \square f_{R}
$$

With reference to the paper [7], the form of the function $f(R)$ is assumed to be ,

$$
f(R)=\xi\left(R+\frac{1}{2} R_{0}\right)+2 \alpha \sqrt{R+R_{0}}
$$

which leads to

$$
R=\frac{\alpha^{2}}{\eta^{2} r^{2}}-R_{0}
$$

where $\alpha, R_{0}$, and $\xi$ are constants. Consequently, the metric function $B(r)$ is obtained for the free parameters $\alpha=\eta$ as,

$$
B(r)=\frac{1}{2}-\frac{m}{r}+\frac{q^{2}}{r^{2}}-\frac{\Lambda_{e f f}}{3} r^{2},
$$

where $m=\frac{-\xi}{3 \eta}, \Lambda_{e f f}=\frac{-R_{0}}{4}$ and $q^{2}=\frac{Q^{2}+P^{2}}{\xi}$. As was explained in [7], due to the constraints on the free parameters, this solution does not admit the Reissner-Nordström (RN)-de Sitter (dS) limit. However, in the limit $\xi=1$ and $P=Q=0$, the solution reduces to the well known global monopole solution reported in [8], which represents a spherically symmetric, non-asymptotically flat solution with a matter field that extends to infinity. Furthermore, this solution can also be considered as a spherically symmetric cloud of cosmic string which gives rise to a deficit angle [9]. Therefore, the solution given in equation (14), is a kind of Einstein-Maxwell extension of the global monopole solution in $f(R)$ gravity. One of the striking effects of the additional fields is the removal of the conical geometry of the global monopole spacetime. The Kretschmann scalar which indicates the formation of curvature singularity is given by

$$
\mathcal{K}=\frac{1}{3} \frac{8 \lambda^{2} r^{8}+4 \lambda r^{6}+3 r^{4}+12 m r^{3}+12 r^{2}\left(3 m^{2}-q^{2}\right)-144 m q^{2} r++168 q^{4}}{r^{8}} .
$$

It is obvious that $r=0$ is a typical central curvature singularity. This is a timelike naked singularity because the behavior of the new radial coordinate defined by $r_{*}=\int \frac{d r}{B(r)}$ is finite when $r \rightarrow 0$. Hence, the new solution obtained in [7] and given in equation (14) is classically a singular spacetime.

Our aim in the next section is to investigate this classically singular spacetime with regard to the quantum mechanical point of view. 


\section{QUANTUM SINGULARITIES}

One of the important predictions of the Einstein's theory of general relativity is the formation of spacetime singularities. In classical general relativity, singularities are defined as the points in which the evolution of timelike or null geodesics is not defined after a proper time. According to the classification of the classical singularities devised by Ellis and Schmidt, scalar curvature singularities are the strongest ones in the sense that the spacetime cannot be extended and all physical quantities, such as the gravitational field, energy density and tidal forces, diverge at the singular point. In black hole spacetimes, the location of the curvature singularity is at $r=0$ and is covered by horizon(s). As long as the singularities are hidden by horizon(s), they do not constitute a threat to the Penrose cosmic censorship hypothesis. However, there are some cases that the singularity is not hidden and hence, it is naked. In the case of naked singularities, further care is required because they violate the cosmic censorship hypothesis. The resolution of the naked singularities stands as one of the most drastic problems in general relativity to be solved.

Naked singularities that occur at $r=0$ are on the very small scales where classical general relativity is expected to be replaced by quantum theory of gravity. In this paper, the occurrence of naked singularities in $f(R)$ gravity will be analyzed through a quantum mechanical point of view. In probing the singularity, quantum test particles/fields obeying the Klein-Gordon, Dirac and Maxwell equations are used. In other words, the singularity will be probed with spin 0 , spin $1 / 2$ and spin 1 fields. The reason for using three different types of field is to clarify whether or not the classical singularity is sensitive to the spin of the fields.

Our analysis will be based on the pioneering work of Wald, which was further developed by HM to probe the classical singularities with quantum test particles obeying the Klein-Gordon equation in static spacetimes having timelike singularities. According to HM, the singular character of the spacetime is defined as the ambiguity in the evolution of the wave functions. That is to say, the singular character is determined in terms of the ambiguity when attempting to find a self-adjoint extension of the operator to the entire Hilbert space. If the extension is unique, it is said that the space is quantum mechanically regular. A brief review now follows:

Consider a static spacetime $\left(M, g_{\mu \nu}\right)$ with a timelike Killing vector field $\xi^{\mu}$. Let $t$ denote the Killing parameter and $\Sigma$ denote a static slice. The Klein-Gordon equation in this space is

$$
\left(\nabla^{\mu} \nabla_{\mu}-M^{2}\right) \psi=0
$$

This equation can be written in the form

$$
\frac{\partial^{2} \psi}{\partial t^{2}}=\sqrt{f} D^{i}\left(\sqrt{f} D_{i} \psi\right)-f M^{2} \psi=-A \psi,
$$

in which $f=-\xi^{\mu} \xi_{\mu}$ and $D_{i}$ is the spatial covariant derivative on $\Sigma$. The Hilbert space $\mathcal{H},\left(L^{2}(\Sigma)\right)$ is the space of square integrable functions on $\Sigma$. The domain of an operator $A, D(A)$, is taken in such a way that it does not enclose the spacetime singularities. An appropriate set is $C_{0}^{\infty}(\Sigma)$, the set of smooth functions with compact support on $\Sigma$. The operator $A$ is real, positive and symmetric; therefore, its self-adjoint extensions always exist. If it has a unique extension $A_{E}$, then $A$ is called essentially self-adjoint [27 29]. Accordingly, the Klein-Gordon equation for a free particle satisfies

$$
i \frac{d \psi}{d t}=\sqrt{A_{E}} \psi
$$

with the solution

$$
\psi(t)=\exp \left[-i t \sqrt{A_{E}}\right] \psi(0)
$$

If $A$ is not essentially self-adjoint, the future time evolution of the wave function (18) is ambiguous. Then the HM criterion defines the spacetime as quantum mechanically singular. However, if there is only a single self-adjoint extension, the operator $A$ is said to be essentially self-adjoint and the quantum evolution described by Eq.(18) is uniquely determined by the initial conditions. According to the HM criterion, this spacetime is said to be quantum mechanically non-singular. In order to determine the number of self-adjoint extensions, the concept of deficiency indices is used. The deficiency subspaces $N_{ \pm}$are defined by ( see Ref. [13] for a detailed mathematical background)

$$
\begin{array}{llll}
N_{+}=\left\{\psi \in D\left(A^{*}\right),\right. & A^{*} \psi=Z_{+} \psi, & \text { Im } \left.Z_{+}>0\right\} & \text { with dimension } n_{+} \\
N_{-}=\left\{\psi \in D\left(A^{*}\right),\right. & A^{*} \psi=Z_{-} \psi, & \text { Im } \left.Z_{-}<0\right\} & \text { with dimension } n_{-}
\end{array}
$$


The dimensions $\left(n_{+}, n_{-}\right)$are the deficiency indices of the operator $A$. The indices $n_{+}\left(n_{-}\right)$are completely independent of the choice of $Z_{+}\left(Z_{-}\right)$depending only on whether or not $Z$ lies in the upper (lower) half complex plane. Generally one takes $Z_{+}=i \lambda$ and $Z_{-}=-i \lambda$, where $\lambda$ is an arbitrary positive constant necessary for dimensional reasons. The determination of deficiency indices is then reduced to counting the number of solutions of $A^{*} \psi=Z \psi ;($ for $\lambda=1)$,

$$
A^{*} \psi \pm i \psi=0
$$

that belong to the Hilbert space $\mathcal{H}$. If there are no square integrable solutions ( i.e. $n_{+}=n_{-}=0$ ), the operator $A$ possesses a unique self-adjoint extension and essentially self-adjoint. Consequently, the way to find a sufficient condition for the operator $A$ to be essentially self-adjoint is to investigate the solutions satisfying Eq. (20) that do not belong to the Hilbert space.

\section{A. Klein-Gordon Fields}

The Klein-Gordon equation for a scalar particle with mass $M$ is given by

$$
\square \psi=g^{-1 / 2} \partial_{\mu}\left[g^{1 / 2} g^{\mu \nu} \partial_{\nu}\right] \psi=M^{2} \psi
$$

For the metric (3), the Klein-Gordon equation becomes

$$
\begin{aligned}
\frac{\partial^{2} \psi}{\partial t^{2}}= & -B(r)\left\{B(r) \frac{\partial^{2} \psi}{\partial r^{2}}+\frac{1}{r^{2}} \frac{\partial^{2} \psi}{\partial \theta^{2}}+\frac{1}{r^{2} \sin ^{2} \theta} \frac{\partial^{2} \psi}{\partial \varphi^{2}}+\frac{\cot \theta}{r^{2}} \frac{\partial \psi}{\partial \theta}+\left(\frac{2 B(r)}{r}+B^{\prime}(r)\right) \frac{\partial \psi}{\partial r}\right\} \\
& +B(r) M^{2} \psi .
\end{aligned}
$$

In analogy with equation (16), the spatial operator $A$ for the massless case is

$$
A=B(r)\left\{B(r) \frac{\partial^{2}}{\partial r^{2}}+\frac{1}{r^{2}} \frac{\partial^{2}}{\partial \theta^{2}}+\frac{1}{r^{2} \sin ^{2} \theta} \frac{\partial^{2}}{\partial \varphi^{2}}+\frac{\cot \theta}{r^{2}} \frac{\partial}{\partial \theta}+\left(\frac{2 B(r)}{r}+B^{\prime}(r)\right) \frac{\partial}{\partial r}\right\}
$$

and the equation to be solved is $\left(A^{*} \pm i\right) \psi=0$.Using separation of variables, $\psi=R(r) Y_{l}^{m}(\theta, \varphi)$, we get the radial portion of equation (20) as

$$
\frac{d^{2} R(r)}{d r^{2}}+\frac{\left(r^{2} B(r)\right)^{\prime}}{r^{2} B(r)} \frac{d R(r)}{d r}+\left(\frac{-l(l+1)}{r^{2} B(r)} \pm \frac{i}{B^{2}(r)}\right) R(r)=0 .
$$

where a prime denotes the derivative with respect to $r$.

\section{The case of $r \rightarrow \infty$}

The case $r \rightarrow \infty$ is topologically different compared to the analysis reported in [21]. In the present problem the geometry is not conical. The approximate metric when $r \rightarrow \infty$ is

$$
d s^{2} \simeq-\left(\frac{R_{0} r^{2}}{12}\right) d t^{2}+\left(\frac{12}{R_{0} r^{2}}\right) d r^{2}+r^{2}\left(d \theta^{2}+\sin ^{2} \theta d \varphi^{2}\right) .
$$

For the above metric, the radial equation (24) becomes,

$$
\frac{d^{2} R(r)}{d r^{2}}+\frac{4}{r} \frac{d R(r)}{d r}=0
$$

whose solution is

$$
R(r)=C_{1}+\frac{C_{2}}{r^{3}},
$$

where $C_{1}$ and $C_{2}$ are arbitrary integration constants. It is clearly observed that the above solution is square integrable as $r \rightarrow \infty$ if and only if $C_{1}=0$. Hence, the asymptotic behavior of $R(r)$ is given by $R(r) \simeq \frac{C_{2}}{r^{3}}$. 


\section{The case of $r \rightarrow 0$}

Near the origin there is a true timelike curvature singularity resulting from the existence of charge. Therefore, the approximate metric near the origin is given by

$$
d s^{2} \simeq-\left(\frac{q^{2}}{r^{2}}\right) d t^{2}+\left(\frac{r^{2}}{q^{2}}\right) d r^{2}+r^{2}\left(d \theta^{2}+\sin ^{2} \theta d \varphi^{2}\right) .
$$

The radial equation (24) for the above metric reduces to

$$
\frac{d^{2} R(r)}{d r^{2}}-\frac{l(l+1)}{q^{2}} R(r)=0
$$

whose solution is

$$
\begin{aligned}
R(r) & =C_{3} e^{\alpha r}+C_{4} e^{-\alpha r} \\
\alpha & =\frac{\sqrt{l(l+1)}}{q}
\end{aligned}
$$

where $C_{3}$ and $C_{4}$ are arbitrary integration constants. The square integrability of the above solution is checked by calculating the squared norm of the above solution in which the function space on each $t=$ constant hypersurface $\Sigma$ is defined as $\mathcal{H}=\{R \mid\|R\|<\infty\}$. The squared norm for the metric (27) is given by,

$$
\|R\|^{2}=\int_{0}^{\mathrm{constant}} \frac{|R(r)|^{2} r^{4}}{q^{2}} d r
$$

Our calculation has revealed that the solution above is always square integrable near $r=0$, even if $l=0$, which corresponds to the $S$-wave solutions.

Consequently, the spatial operator $A$ has deficiency indices $n_{+}=n_{-}=1$, and it is not essentially self-adjoint. Hence, the classical singularity at $r=0$ remains quantum mechanically singular when probed with fields obeying the Klein-Gordon equation.

\section{B. Maxwell fields}

The Newman-Penrose formalism will be used to find the source-free Maxwell fields propagating in the space of $f(R)$ gravity. Let us note that the signature of the metric (3) is changed to -2 in order to use the source-free Maxwell equations in Newman-Penrose formalism. Thus, the metric (3) is rewritten as,

$$
d s^{2}=B(r) d t^{2}-\frac{d r^{2}}{B(r)}-r^{2}\left(d \theta^{2}+\sin ^{2} \theta d \varphi^{2}\right) .
$$

The four coupled source-free Maxwell equations for electromagnetic fields in the Newman-Penrose formalism is given by

$$
\begin{aligned}
D \phi_{1}-\bar{\delta} \phi_{0} & =(\pi-2 \alpha) \phi_{0}+2 \rho \phi_{1}-\kappa \phi_{2}, \\
\delta \phi_{2}-\Delta \phi_{1} & =-\nu \phi_{0}+2 \mu \phi_{1}+(\tau-2 \beta) \phi_{2}, \\
\delta \phi_{1}-\Delta \phi_{0} & =(\mu-2 \gamma) \phi_{0}+2 \tau \phi_{1}-\sigma \phi_{2}, \\
D \phi_{2}-\bar{\delta} \phi_{1} & =-\lambda \phi_{0}+2 \pi \phi_{1}+(\rho-2 \epsilon) \phi_{2},
\end{aligned}
$$

where $B(r)$ is the metric function given in Eq.(14), $\phi_{0}, \phi_{1}$ and $\phi_{2}$ are the Maxwell spinors, $\epsilon, \rho, \pi, \alpha, \mu, \gamma, \beta$ and $\tau$ are the spin coefficients to be found and the bar denotes complex conjugation. The null tetrad vectors for the metric (31) are defined by

$$
\begin{aligned}
l^{a} & =\left(\frac{1}{B(r)}, 1,0,0\right), \\
n^{a} & =\left(\frac{1}{2},-\frac{B(r)}{2}, 0,0\right), \\
m^{a} & =\frac{1}{\sqrt{2}}\left(0,0, \frac{1}{r}, \frac{i}{r \sin \theta}\right) .
\end{aligned}
$$


The directional derivatives in the Maxwell's equations are defined by $D=l^{a} \partial_{a}, \Delta=n^{a} \partial_{a}$ and $\delta=m^{a} \partial_{a}$. We define operators in the following way

$$
\begin{aligned}
& \mathbf{D}_{0}=D \\
& \mathbf{D}_{0}^{\dagger}=-\frac{2}{B(r)} \Delta, \\
& \mathbf{L}_{0}^{\dagger}=\sqrt{2} r \delta \text { and } \mathbf{L}_{1}^{\dagger}=\mathbf{L}_{0}^{\dagger}+\frac{\cot \theta}{2} \\
& \mathbf{L}_{0}=\sqrt{2} r \bar{\delta} \text { and } \mathbf{L}_{1}=\mathbf{L}_{0}+\frac{\cot \theta}{2} .
\end{aligned}
$$

The non-zero spin coefficients are

$$
\mu=-\frac{1}{r} \frac{B(r)}{2}, \quad \rho=-\frac{1}{r}, \quad \gamma=\frac{1}{4} B^{\prime}(r), \quad \beta=-\alpha=\frac{1}{2 \sqrt{2}} \frac{\cot \theta}{r} .
$$

The Maxwell spinors are defined by [30]

$$
\begin{aligned}
\phi_{0} & =F_{13}=F_{\mu \nu} l^{\mu} m^{\nu} \\
\phi_{1} & =\frac{1}{2}\left(F_{12}+F_{43}\right)=\frac{1}{2} F_{\mu \nu}\left(l^{\mu} n^{\nu}+\bar{m}^{\mu} m^{\nu}\right), \\
\phi_{2} & =F_{42}=F_{\mu \nu} \bar{m}^{\mu} n^{\nu},
\end{aligned}
$$

where $F_{i j}(i, j=1,2,3,4)$ and $F_{\mu \nu}(\mu, \nu=0,1,2,3)$ are the components of the Maxwell tensor in the tetrad and tensor bases, respectively. Substituting Eq.(34) into the Maxwell's equations together with non-zero spin coefficients, the Maxwell equations become

$$
\begin{gathered}
\left(\mathbf{D}_{0}+\frac{2}{r}\right) \phi_{1}-\frac{1}{r \sqrt{2}} \mathbf{L}_{1} \phi_{0}=0, \\
\left(\mathbf{D}_{0}+\frac{1}{r}\right) \phi_{2}-\frac{1}{r \sqrt{2}} \mathbf{L}_{0} \phi_{1}=0, \\
\frac{B(r)}{2}\left(\mathbf{D}_{0}^{\dagger}+\frac{B^{\prime}(r)}{B(r)}+\frac{1}{r}\right) \phi_{0}+\frac{1}{r \sqrt{2}} \mathbf{L}_{0}^{\dagger} \phi_{1}=0, \\
\frac{B(r)}{2}\left(\mathbf{D}_{0}^{\dagger}+\frac{2}{r}\right) \phi_{1}+\frac{1}{r \sqrt{2}} \mathbf{L}_{1}^{\dagger} \phi_{2}=0 .
\end{gathered}
$$

The equations above will become more tractable if the variables are changed to

$$
\Phi_{0}=\phi_{0} e^{i k t}, \quad \Phi_{1}=\sqrt{2} r \phi_{1} e^{i k t}, \quad \Phi_{2}=2 r^{2} \phi_{2} e^{i k t}
$$

Then we have

$$
\begin{gathered}
\left(\mathbf{D}_{0}+\frac{1}{r}\right) \Phi_{1}-\mathbf{L}_{1} \Phi_{0}=0, \\
\left(\mathbf{D}_{0}-\frac{1}{r}\right) \Phi_{2}-\mathbf{L}_{0} \Phi_{1}=0, \\
r^{2} B(r)\left(\mathbf{D}_{0}^{\dagger}+\frac{B^{\prime}(r)}{B(r)}+\frac{1}{r}\right) \Phi_{0}+\mathbf{L}_{0}^{\dagger} \Phi_{1}=0, \\
r^{2} B(r)\left(\mathbf{D}_{0}^{\dagger}+\frac{1}{r}\right) \Phi_{1}+\mathbf{L}_{1}^{\dagger} \Phi_{2}=0 .
\end{gathered}
$$


The commutativity of the operators $\mathbf{L}$ and $\mathbf{D}$ enables us to eliminate each $\Phi_{i}$ from above equations, and hence we have

$$
\begin{gathered}
{\left[\mathbf{L}_{0}^{\dagger} \mathbf{L}_{1}+r^{2} B(r)\left(\mathbf{D}_{0}+\frac{B^{\prime}(r)}{B(r)}+\frac{3}{r}\right)\left(\mathbf{D}_{0}^{\dagger}+\frac{B^{\prime}(r)}{B(r)}+\frac{1}{r}\right)\right] \Phi_{0}(r, \theta)=0,} \\
{\left[\mathbf{L}_{0} \mathbf{L}_{1}^{\dagger}+r^{2} B(r)\left(\mathbf{D}_{0}^{\dagger}+\frac{1}{r}\right)\left(\mathbf{D}_{0}-\frac{1}{r}\right)\right] \Phi_{2}(r, \theta)=0,} \\
{\left[\mathbf{L}_{1} \mathbf{L}_{0}^{\dagger}+r^{2} B(r)\left(\mathbf{D}_{0}^{\dagger}+\frac{B^{\prime}(r)}{B(r)}+\frac{1}{r}\right)\left(\mathbf{D}_{0}+\frac{1}{r}\right)\right] \Phi_{1}(r, \theta)=0 .}
\end{gathered}
$$

The variables $r$ and $\theta$ can be separated by assuming a separable solution in the form of

$$
\Phi_{0}(r, \theta)=f_{0}(r) \Theta_{0}(\theta), \quad \Phi_{1}(r, \theta)=f_{1}(r) \Theta_{1}(\theta), \quad \Phi_{2}(r, \theta)=f_{2}(r) \Theta_{2}(\theta) .
$$

The separation constants for Eq. (45) and Eq. (46) are the same, because $\mathbf{L}_{n}=-\mathbf{L}_{n}^{\dagger}(\pi-\theta)$, or, in other words, the operator $\mathbf{L}_{0}^{\dagger} \mathbf{L}_{1}$ acting on $\Theta_{0}(\theta)$ is the same as the operator $\mathbf{L}_{0} \mathbf{L}_{1}^{\dagger}$ acting on $\Theta_{2}(\theta)$ if we replace $\theta$ by $\pi-\theta$. However, for Eq. (47) we will assume another separation constant. Furthermore, by defining $R_{0}(r)=\frac{f_{0}(r)}{r B(r)}, R_{1}(r)=\frac{f_{1}(r)}{r}$ and $R_{2}(r)=\frac{f_{2}(r)}{r}$, the radial equations can be written as

$$
\begin{aligned}
& f_{0}^{\prime \prime}(r)+\frac{2}{r} f_{0}^{\prime}(r)+ \\
& {\left[-i \omega\left(\frac{2}{r B(r)}-\frac{B^{\prime}(r)}{B^{2}(r)}\right)+\frac{\omega^{2}}{B^{2}(r)}-\frac{\epsilon^{2}}{r^{2} B(r)}\right] f_{0}(r)=0} \\
& f_{2}^{\prime \prime}(r)-\frac{2}{r} f_{2}^{\prime}(r)+ \\
& {\left[i \omega\left(\frac{2}{r B(r)}-\frac{B^{\prime}(r)}{B^{2}(r)}\right)+\frac{\omega^{2}}{B^{2}(r)}-\frac{\epsilon^{2}}{r^{2} B(r)}\right] f_{2}(r)=0,} \\
& f_{1}^{\prime \prime}(r)+\frac{B^{\prime}(r)}{B(r)} f_{1}^{\prime}(r)+ \\
& {\left[\frac{\omega^{2}}{B^{2}(r)}-\frac{\eta^{2}}{r^{2} B(r)}\right] f_{1}(r)=0,}
\end{aligned}
$$

where $\epsilon$ and $\eta$ are the separability constants.

\section{The case $r \rightarrow \infty$}

For the case $r \rightarrow \infty$, the corresponding metric is given in Eq.(25). Hence, the radial parts of the Maxwell equations, (48), (49) and (50), become

$$
\begin{aligned}
& f_{j}^{\prime \prime}(r)+\frac{2}{r} f_{j}^{\prime}(r)=0, \quad j=0,1 \\
& f_{2}^{\prime \prime}(r)-\frac{2}{r} f_{2}^{\prime}(r)=0
\end{aligned}
$$

Thus, the solutions in the asymptotic case are

$$
\begin{aligned}
& R_{j}(r)=C_{1}+\frac{C_{2}}{r}, \quad j=0,1 \\
& R_{2}(r)=C_{3}+\frac{C_{4}}{r^{3}}
\end{aligned}
$$

in which $C_{i}$ are integration constants. The solution above is square integrable if $C_{1}=C_{3}=0$. Therefore, the asymptotic form of the solutions behaves as $R_{j}(r) \sim \frac{C_{2}}{r}, \quad j=0,1$ and $R_{2}(r) \sim \frac{C_{4}}{r^{3}}$. 


\section{The case $r \rightarrow 0$}

The metric near $r \rightarrow 0$ is given in Eq.(27). Hence, the radial parts of the Maxwell equations (48), (49) and (50) for this case are given by

$$
\begin{aligned}
& R_{j}^{\prime \prime}(r)-\frac{2}{r} R_{j}^{\prime}(r)-\frac{\alpha^{2}}{q^{2}} R_{j}(r)=0, j=1,2 \\
& R_{0}^{\prime \prime}(r)+\frac{2}{r} R_{0}^{\prime}(r)-\frac{\eta^{2}}{q^{2}} R_{0}(r)=0
\end{aligned}
$$

whose solutions are obtained as,

$$
\begin{aligned}
R_{j}(r) & =C_{3} e^{\frac{\alpha}{q} r}(\alpha r-1)+C_{4} e^{-\frac{\alpha}{q} r}(\alpha r+1), \quad j=1,2 \\
R_{0}(r) & =\frac{C_{5}}{r} \sinh \left(\frac{\eta}{q} r\right)+\frac{C_{6}}{r} \cosh \left(\frac{\eta}{q} r\right)
\end{aligned}
$$

where $C_{i}$ are constants. The above solution is checked for square integrability. Calculations have revealed that

$$
\left\|R_{i}\right\|^{2}=\int_{0}^{\text {constant }} \frac{\left|R_{i}(r)\right|^{2} r^{4}}{q^{2}} d r<\infty
$$

which indicates that the obtained solutions are square integrable. The definition of the quantum singularity for Maxwell fields will be the same as for the Klein-Gordon fields. Here, since we have three equations governing the dynamics of the photon waves, the unique self-adjoint extension condition on the spatial part of the Maxwell operator should be examined for each of the three equations. As a result, the occurrence of the naked singularity in $f(R)$ gravity is quantum mechanically singular if it is probed with photon waves.

\section{Dirac Fields}

The Newman-Penrose formalism will also be used here to find the massless Dirac fields (fermions) propagating in the space of $f(R)$-gravity. The Chandrasekhar-Dirac (CD) equations in the Newman-Penrose formalism are given by

$$
\begin{aligned}
(D+\epsilon-\rho) F_{1}+(\bar{\delta}+\pi-\alpha) F_{2} & =0 \\
(\Delta+\mu-\gamma) F_{2}+(\delta+\beta-\tau) F_{1} & =0 \\
(D+\bar{\epsilon}-\bar{\rho}) G_{2}-(\delta+\bar{\pi}-\bar{\alpha}) G_{1} & =0 \\
(\Delta+\bar{\mu}-\bar{\gamma}) G_{1}-(\bar{\delta}+\bar{\beta}-\bar{\tau}) G_{2} & =0
\end{aligned}
$$

where $F_{1}, F_{2}, G_{1}$ and $G_{2}$ are the components of the wave function, $\epsilon, \rho, \pi, \alpha, \mu, \gamma, \beta$ and $\tau$ are the spin coefficients to be found. The non-zero spin coefficients are given in Eq.(35). The directional derivatives in the CD equations are the same as in the Maxwell equations. Substituting non-zero spin coefficients and the definitions of the operators given in Eq.(34) into the CD equations leads to

$$
\begin{gathered}
\left(\mathbf{D}_{0}+\frac{1}{r}\right) F_{1}+\frac{1}{r \sqrt{2}} \mathbf{L}_{1} F_{2}=0, \\
-\frac{B(r)}{2}\left(\mathbf{D}_{0}^{\dagger}+\frac{B^{\prime}(r)}{2 B(r)}+\frac{1}{r}\right) F_{2}+\frac{1}{r \sqrt{2}} \mathbf{L}_{1}^{\dagger} F_{1}=0, \\
\left(\mathbf{D}_{0}+\frac{1}{r}\right) G_{2}-\frac{1}{r \sqrt{2}} \mathbf{L}_{1}^{\dagger} G_{1}=0, \\
\frac{B(r)}{2}\left(\mathbf{D}_{0}^{\dagger}+\frac{B^{\prime}(r)}{2 B(r)}+\frac{1}{r}\right) G_{1}+\frac{1}{r \sqrt{2}} \mathbf{L}_{1} G_{2}=0 .
\end{gathered}
$$


For the solution of the CD equations, we assume a separable solution in the form of

$$
\begin{aligned}
F_{1} & =f_{1}(r) Y_{1}(\theta) e^{i(k t+m \varphi)}, \\
F_{2} & =f_{2}(r) Y_{2}(\theta) e^{i(k t+m \varphi)}, \\
G_{1} & =g_{1}(r) Y_{3}(\theta) e^{i(k t+m \varphi)}, \\
G_{2} & =g_{2}(r) Y_{4}(\theta) e^{i(k t+m \varphi)},
\end{aligned}
$$

where $m$ is the azimuthal quantum number and $k$ is the frequency of the Dirac fields, which is assumed to be positive and real .Since $\left\{f_{1}, f_{2}, g_{1}, g_{2}\right\}$ and $\left\{Y_{1}, Y_{2}, Y_{3}, Y_{4}\right\}$ are functions of $r$ and $\theta$, respectively, by substituting Eq.(61) into Eq.(60) and applying the assumptions given by

$$
\begin{aligned}
& f_{1}(r)=g_{2}(r) \quad \text { and } \quad f_{2}(r)=g_{1}(r), \\
& Y_{1}(\theta)=Y_{3}(\theta) \quad \text { and } \quad Y_{2}(\theta)=Y_{4}(\theta),
\end{aligned}
$$

the Dirac equations transform into Eq.(64). In order to solve the radial equations, the separation constant $\lambda$ should be defined. This is achieved by using the angular equations. In fact, it is already known from the literature that the separation constant can be expressed in terms of the spin-weighted spheroidal harmonics. The radial parts of the Dirac equations become

$$
\begin{gathered}
\left(\mathbf{D}_{0}+\frac{1}{r}\right) f_{1}(r)=\frac{\lambda}{r \sqrt{2}} f_{2}(r), \\
\frac{B(r)}{2}\left(\mathbf{D}_{0}^{\dagger}+\frac{B^{\prime}(r)}{2 B(r)}+\frac{1}{r}\right) f_{2}(r)=\frac{\lambda}{r \sqrt{2}} f_{1}(r) .
\end{gathered}
$$

We further assume that

$$
\begin{aligned}
& f_{1}(r)=\frac{\Psi_{1}(r)}{r}, \\
& f_{2}(r)=\frac{\Psi_{2}(r)}{r},
\end{aligned}
$$

then Eq.(64) transforms into,

$$
\begin{gathered}
\mathbf{D}_{0} \Psi_{1}=\frac{\lambda}{r \sqrt{2}} \Psi_{2}, \\
\frac{B(r)}{2}\left(\mathbf{D}_{0}^{\dagger}+\frac{B^{\prime}(r)}{2 B(r)}\right) \Psi_{2}=\frac{\lambda}{r \sqrt{2}} \Psi_{1} .
\end{gathered}
$$

Note that $\sqrt{\frac{B(r)}{2}} \mathbf{D}_{0}^{\dagger} \sqrt{\frac{B(r)}{2}}=\mathbf{D}_{0}^{\dagger}+\frac{B^{\prime}(r)}{2 B(r)}+\frac{1}{r}$, and using this together with the new functions

$$
\begin{aligned}
& R_{1}(r)=\Psi_{1}(r), \\
& R_{2}(r)=\sqrt{\frac{B(r)}{2}} \Psi_{2}(r),
\end{aligned}
$$

and defining the tortoise coordinate $r_{*}$ as

$$
\frac{d}{d r_{*}}=B \frac{d}{d r}
$$

Eqs.(65) become

$$
\begin{aligned}
& \left(\frac{d}{d r_{*}}+i k\right) R_{1}=\frac{\sqrt{B} \lambda}{r} R_{2}, \\
& \left(\frac{d}{d r_{*}}-i k\right) R_{2}=\frac{\sqrt{B} \lambda}{r} R_{1}
\end{aligned}
$$


In order to write Eq.(67) in a more compact form, we combine the solutions in the following way:

$$
\begin{aligned}
& Z_{+}=R_{1}+R_{2}, \\
& Z_{-}=R_{2}-R_{1} .
\end{aligned}
$$

After doing some calculations we end up with a pair of one-dimensional Schrödinger-like wave equations with effective potentials,

$$
\begin{gathered}
\left(\frac{d^{2}}{d r_{*}^{2}}+k^{2}\right) Z_{ \pm}=V_{ \pm} Z_{ \pm}, \\
V_{ \pm}=\left[\frac{B \lambda^{2}}{r^{2}} \pm \lambda \frac{d}{d r_{*}}\left(\frac{\sqrt{B}}{r}\right)\right] .
\end{gathered}
$$

In analogy with equation (16), the radial operator $A$ for the Dirac equations can be written as,

$$
A=-\frac{d^{2}}{d r_{*}^{2}}+V_{ \pm}
$$

If we write the above operator in terms of the usual coordinates $r$ by using Eq.(66), we have

$$
A=-\frac{d^{2}}{d r^{2}}-\frac{B^{\prime}}{B} \frac{d}{d r}+\frac{1}{B^{2}}\left[\frac{B \lambda^{2}}{r^{2}} \pm \lambda B \frac{d}{d r}\left(\frac{\sqrt{B}}{r}\right)\right],
$$

Our aim now is to show whether this radial part of the Dirac operator is essentially self-adjoint or not. This will be achieved by considering Eq.(20) and counting the number of solutions that do not belong to Hilbert space. Hence, Eq.(20) becomes

$$
\left(\frac{d^{2}}{d r^{2}}+\frac{B^{\prime}}{B} \frac{d}{d r}-\frac{1}{B^{2}}\left[\frac{B \lambda^{2}}{r^{2}} \pm \lambda B \frac{d}{d r}\left(\frac{\sqrt{B}}{r}\right)\right] \mp i\right) \psi(r)=0
$$

For the asymptotic case, $r \rightarrow \infty$, the above equation transforms to

$$
\frac{d^{2} \psi}{d r^{2}}+\frac{2}{r} \frac{d \psi}{d r}=0
$$

whose solution is

$$
\psi(r)=C_{1}+\frac{C_{2}}{r} .
$$

Clearly the solution is square integrable if $C_{1}=0$. Hence, the solution is asmptotically well behaved. Near $r \rightarrow 0$, Eq.(71) becomes

$$
\begin{gathered}
\frac{d^{2} \psi}{d r^{2}}-\frac{2}{r} \frac{d \psi}{d r}+\frac{\sigma}{r^{3}} \psi=0, \\
\sigma=\mp 2 \lambda q,
\end{gathered}
$$

whose solution is given by

$$
\psi(r)=\left(\frac{4 \sigma}{x^{2}}\right)^{\frac{3}{2}}\left\{C_{3} J_{3}(x)+C_{4} N_{3}(x)\right\},
$$

where $J_{3}(x)$ and $N_{3}(x)$ are Bessel functions of the first and second kind, and $x=2 \sqrt{\frac{\sigma}{r}}$. As $r \rightarrow 0$, we have $x \rightarrow \infty$. The behavior of the Bessel functions for real $\nu \geq 0$ as $x \rightarrow \infty$ is given by

$$
\begin{aligned}
J_{\nu}(x) & \simeq \sqrt{\frac{2}{\pi x}} \cos \left(x-\frac{\nu \pi}{2}-\frac{\pi}{4}\right), \\
N_{\nu}(x) & \simeq \sqrt{\frac{2}{\pi x}} \sin \left(x-\frac{\nu \pi}{2}-\frac{\pi}{4}\right) ;
\end{aligned}
$$


thus the Bessel functions asymptotically behave as $J_{3}(x) \sim \sqrt{\frac{2}{\pi x}} \cos \left(x-\frac{7 \pi}{4}\right)$ and $N_{3}(x) \sim \sqrt{\frac{2}{\pi x}} \sin \left(x-\frac{7 \pi}{4}\right)$. Checking for the square integrability has revealed that both solutions are square integrable. Hence, the radial operator of the Dirac field fails to satisfy a unique self-adjoint extension condition. As a result, the occurrence of the timelike naked singularity in the context of $f(R)$ gravity remains singular from the quantum mechanical point of view if it is probed with fermions.

\section{CONCLUSION}

In this paper, the formation of the naked singularity in the context of a model of $f(R)$ gravity is investigated within the framework of quantum mechanics, by probing the singularity with the quantum fields obeying the Klein-Gordon, Maxwell and Dirac equations. We have investigated the essential self-adjointness of the spatial part of the wave operator $A$ in the natural Hilbert space of quantum mechanics which is a linear function space with square integrability. Our analysis has shown that the timelike naked curvature singularity remains quantum mechanically singular against the propagation of the aforementioned quantum fields. Another notable outcome of our analysis is that the spin of the fields is not effective in healing of the naked singularity for the considered model of the $f(R)$ gravity spacetime.

Another alternative function space for analyzing the singularity in this context is to use the Sobelov space instead of the natural Hilbert space [13]. The Analysis in Sobelov space entails square integrability both of the wave function and its derivative. Although the details are not given in this study, the analysis using the Sobelov space has revealed that irrespective of the spin structure of the fields used to probe the singularity, the model considered of $f(R)$ gravity spacetime remains quantum mechanically singular.

Hence, the generic conclusion that has emerged from our analysis is that in the model considered of $f(R)$ gravity, the formation of a timelike naked singularity is quantum mechanically singular.

It will be interesting for future research to extend the quantum singularity analysis in other ETG models. Furthermore, it will be a great achievement if the criterion proposed by HM is extended to stationary metrics. Although the preliminary work in this direction is considered in [31], the formulation has not been fully completed.

[1] S. Nojiri and S.D. Odintsov, Phys. Rep. 505, 59 (2011).

[2] T. Clifton and J. D. Barrow, Phys. Rev. D 72, 103005 (2005).

[3] T. Multamaki and I. Vilja, Phys. Rev. D 76, 064021 (2007).

[4] M. D. Seifert, Phys. Rev. D 76, 064002 (2007).

[5] L. Hollenstein and F. S. N. Lobo, Phys. Rev. D 78, 124007 (2008).

[6] A. de la Cruz-Dombriz, A. Dobado and A. L. Maroto, Phys. Rev. D 80, 124011 (2009).

[7] S. Habib Mazharimousavi, M. Halilsoy and T. Tahamtan, Eur. Phys. J. C, 72, 1851 (2012).

[8] M. Barriola and A. Vilenkin, Phys. Rev. Lett. 63, 341 (1989).

[9] P. S. Letelier, Phys. Rev. D 20, 1294 (1979).

[10] S. Capozziello and M. De Laurentis, Phys. Rep. 509, 167 (2011).

[11] R. M. Wald, J. Math. Phys. (N.Y.) 21, 2082 (1980).

[12] G. T. Horowitz and D. Marolf, Phys. Rev. D 52, 5670 (1995).

13] A. Ishibashi and A. Hosoya, Phys. Rev. D 60, 104028 (1999).

[14] D. A. Konkowski and T. M. Helliwell, Gen. Rel. and Grav. 33, 1131, (2001).

[15] T. M. Helliwell, D. A. Konkowski and V. Arndt, Gen. Rel. and Grav. 35, 79, (2003).

[16] D. A. Konkowski, T. M. Helliwell and C. Wieland, Class. Quantum Grav. 21, 265 (2004).

[17] D. A. Konkowski, C. Reese, T. M. Helliwell and C. Wieland, " Classical and Quantum Singularities of Levi-Civita Spacetimes with and without a Cosmological Constant", in Procedings of the Workshop on the Dynamics and Thermodynamics of Black holes and Naked Singularities, ed. L.Fatibene, M. Francaviglia, R. Giambo and G. Megli, 2004.

[18] D. A. Konkowski and T. M. Helliwell, International Journal of Modern Physics A, Vol.26, No.22, 3878-3888 (2011).

[19] J. P. M. Pitelli and P. S. Letelier, J. Math. Phys. 48, 092501, (2007).

[20] J. P. M. Pitelli and P. S. Letelier, Phys. Rev. D 77, 124030 (2008).

[21] J. P. M. Pitelli and P. S. Letelier, Phys. Rev. D 80, 104035 (2009).

[22] P. S. Letelier and J. P. M. Pitelli, Phys. Rev. D 82, 104046 (2010).

[23] O. Unver and O. Gurtug, Phys. Rev. D 82, 084016 (2010).

[24] S. Habib Mazharimousavi, O. Gurtug and M. Halilsoy, Int. J. Mod. Phys. D18, 2061-2082, (2009).

[25] S. Habib Mazharimousavi, M. Halilsoy, I. Sakalli and O. Gurtug, Class. Quant. Grav. 27, 105005, (2010).

[26] S. Habib Mazharimousavi, O. Gurtug, M. Halilsoy and O. Unver, Phys. Rev. D 84, 124021 (2011).

[27] M. Reed and B. Simon, Functional Analysis, (Academic Press, New York, 1980).

[28] M. Reed and B. Simon, Fourier Analysis and Self-Adjointness, (Academic Press, New York, 1975). 
[29] R. D. Richtmyer, Principles of Advanced Mathematical Physics, (Springer, New York, 1978).

[30] S. Chandrasekhar, The Mathematical Theory of Black Holes, (Oxford University Press, 1992).

[31] I. Seggev, Class. Quant. Grav. 21, 2651, (2004). 